\title{
Pharmacogenomics in Radionuclide Therapy: Impact on Response to Theranostics
}

\author{
Andrew M. Scott ${ }^{1}$ and Lisa Bodei ${ }^{2}$ \\ ${ }^{I}$ Department of Molecular Imaging and Therapy at Austin Health, Olivia Newton-John Cancer Research Institute and School of \\ Cancer Medicine at La Trobe University, and Department of Medicine at University of Melbourne, Victoria, Australia; and \\ ${ }^{2}$ Molecular Imaging and Therapy Service, Department of Radiology, Memorial Sloan Kettering Cancer Center, New York, New York
}

$\mathbf{T}$ treatment is a crucial issue in modern medicine and may be due to a range of pharmacologic, metabolic, and genomic factors (1). Through deeper understanding of the impact of genetic differences between patients, it has become clear that predisposition to developing cancer, and response to drugs and other therapies, including radiation therapy, may be explained in many patients by underlying changes in germline DNA (1). In some circumstances, somatic mutations or alterations in gene expression that may be present in tumor cells can impact target expression, drug sensitivity, and response to radiation $(2,3)$. Tumor pharmacogenomics is the study of how the genome influences a patient's response to different cancer drug treatments. These genomic changes may also have an impact on successful radionuclide therapy, and understanding the role of pharmacogenomics in selecting patients for treatment is essential in developing new radiopharmaceutical therapies in cancer patients.

\section{GERMLINE AND SOMATIC MUTATIONS IN CANCER}

Pharmacogenomics in cancer involves 2 genomes, the germline and the tumor. The germline genome involves inherited genetic variations, and the tumor genome considers any somatic mutations that accumulate as a cancer evolves. There are several well-established germline variants that may influence drug metabolism and pharmacokinetics in cancer patients, particularly UGT1A1 (irinotecan), CYP2D6 (tamoxifen), and TPMT (mercaptopurine) (1). Some germline mutations are associated with predisposition to cancer, such as BRCA1/BRCA2 (breast and ovarian cancer) and MLH1 (colon cancer). Somatic mutations are frequently found in cancers, may drive the phenotype of tumors, and influence response to therapy. For example, mutations in EGFR may affect sensitivity to tyrosine kinase inhibitors, and variants of androgen and estrogen receptors may influence hormonal therapy in prostate and breast cancer patients (1). Specific somatic mutations may be preferentially targeted by purposed drugs, such as BRAFV600E mutations in malignant melanoma (1). In this context, knowledge of the role of germline and somatic mutations can have major implications on the selection of therapy and prediction of potential toxicity in

Received Nov. 4, 2020; revision accepted Dec. 16, 2020.

For correspondence or reprints, contact Andrew M. Scott (andrew.scott@ austin.org.au).

Published online Dec. 31, 2020.

COPYRIGHT (C) 2021 by the Society of Nuclear Medicine and Molecular Imaging. DOI: 10.2967/jnumed.120.254995 individual patients. Thus, mutation testing is increasingly important in the standard of care and in cancer drug development.

\section{GENOMIC MUTATIONS AND RADIATION}

Radiation therapy is associated with induction of DNA singlestrand breaks, double-strand breaks, DNA base damage, and indirect damage from oxygenated free radicals, leading to cell death (2). Mutations in DNA repair genes may therefore play an important role in both the development of cancer and the response to radiotherapy. It has been well established that germline variants may be associated with toxicity to radiotherapy, particularly DNA damage response genes such as ATM (2). Germline mutations in both ATM and MRE11 have been shown to be linked to increased responsiveness to radiotherapy.

\section{PHARMACOGENOMICS IN RADIONUCLIDE THERAPY}

The use of $\beta$ - and $\alpha$-emitting radionuclide therapies has been a key component in nuclear medicine practice for decades, particularly in the last 20 years by the development of peptide-receptor radionuclide therapy (PRRT) and, recently, prostate-specific membrane antigen (PSMA) radioligand therapy. The development of these therapeutic approaches has focused mainly on patient selection for treatment based on expression of the target in the tumor, namely a surrogate for the absorbed dose, but has, so far, neglected tumor and tissue radiosensitivity, which is the other term of the radiation effect equation. Despite these improved techniques for effective delivery of radionuclide to cancer cells, and strategies for reducing toxicity, there are still many patients who do not respond to treatment. The role of germline variants and somatic mutations in tumors in therapeutic response to radionuclide therapy is an area of increasing relevance for patient selection and monitoring of response. How can we use pharmacogenomics to improve our approaches to treatment of cancer patients with radionuclides?

\section{THYROID CANCER}

The treatment of well-differentiated thyroid cancer with surgery and, in appropriate patients, ${ }^{131} \mathrm{I}$ therapy has been the mainstay of patient management for over 40 years. However, there are some patients in whom ${ }^{131}$ I therapy is not successful or in whom radioiodine uptake is not evident in some metastatic lesions, and in these patients downregulation of the NaI symporter in tumor cells may be responsible for reduced efficacy. Redifferentiation therapy may induce $\mathrm{NaI}$ expression and restore responsiveness to ${ }^{131} \mathrm{I}$ therapy, 
and studies have shown that tyrosine kinase inhibitors against $N R A S$ and BRAFV600E mutations in tumors can be successfully used to allow effective ${ }^{131}$ I treatment to be given in such patients (4).

\section{NEUROENDOCRINE TUMORS}

Well-differentiated neuroendocrine tumors (NETs) generally exhibit low mutation burdens. Mutations are infrequent in smallintestine types, mainly involving $C D K N 1 B(<10 \%)$, whereas other mutations, such as $B R A F, K R A S$, and TP53, are even rarer. Germline mutations of MUTYH and IPMK are sporadic. Pancreatic NET mutations are more common, mainly of MEN1 (35\%-50\%), DAXX (20\%), and ATRX $(10 \%)$, although other genes, such as DNA damage repair or negative regulators, may be involved. Bronchopulmonary NETs exhibit mutations of histone modifiers in about $40 \%$ of cases, including MEN1 and TP53. Many of these mutations (e.g., $M E N 1, T P 53, D A X X$, and $A T M$ ) have prognostic significance, and none so far has been specifically associated with response to PRRT.

Tumor gene expression profiling has revealed that NET subtypes have characteristic features. Downregulation of $T T F 1$ was the only demonstrated association with a poor response to PRRT in bronchopulmonary NETs (3), although this finding was likely a prognostic characteristic, namely independent from the treatment applied. Multigene signature analyses of circulating messenger RNA have been introduced as liquid biopsies. High expression of growth-factor signaling and metabolism genes was noted to be associated with absence of progression on PRRT. Integration of gene expression with Ki-67 grading forms the basis for a PRRT-prediction quotient that functions as a predictive biomarker with 95\% accuracy in 3 independent prospective cohorts. Notably, the PRRT-prediction quotient could not predict the effect of other therapies, therefore representing a specific radiation sensitivity signature for PRRT. Although several single-nucleotide polymorphisms and gene expression profiles have been associated with radiation injury, no specific tool exists for PRRT toxicity yet (5).

\section{PROSTATE CANCER}

There are inherited germline mutations in DNA-repair genes that are linked to more aggressive prostate cancer, including $B R C A 2$, $C H E K 2$, and $A T M$, although the frequency of these mutations is low. Germline mutations are also associated with responsiveness to antiandrogen therapy (e.g., SLCO2B1) (1). Somatic mutations in the androgen receptor (including splice variants) and p53 are the most common seen in prostate cancer, although mutations in DNA-repair genes may also be found in up to $23 \%$ of metastatic castration-resistant prostate cancer.

There are emerging reports of the impact of germline and somatic mutations in patients with metastatic castration-resistant prostate cancer treated with PSMA radioligand therapy. In one study, germline mutations in $C H E K 2$ but not in other radiosensitizer genes (e.g., $F A N C A, B R C A 1$, and $A T R$ ) was associated with prostate-specific the antigen response to ${ }^{177} \mathrm{Lu}$-PSMA (6). In a study of patients treated with ${ }^{225}$ Ac-PSMA, somatic mutations (e.g., TP53, CHEK2, and $A T M$ ) were identified in biopsies of metastatic lesions that did not respond to therapy despite high PSMA uptake on screening PSMA PET, suggesting a mechanism of resistance to therapy (7). A case report of a patient who had a poor response to ${ }^{177} \mathrm{Lu}-$ PSMA therapy despite high uptake on PSMA PET, but who was found to have a germline $B R C A 2$ mutation present and subsequently responded to poly(adenosine diphosphate)-ribose polymerase
(PARP) inhibitor therapy, confirms the importance of mutation testing of patients with a poor response to PSMA radioligand therapy (8). Recent data evaluating plasma androgen receptor gene expression in metastatic castration-resistant prostate cancer revealed that high gene levels identified resistance to ${ }^{177}$ Lu-PSMA-617, which is likely a prognostic feature (9).

The role of DNA-repair somatic mutations in prostate cancer has also been exploited by the use of PARP inhibitors, which inhibit DNA repair. The PARP inhibitors olaparib and rucaparib were recently approved for treating patients with metastatic castrationresistant prostate cancer when germline or somatic DNA repair mutations are present. Multiple clinical trials of PARP inhibitors with radiotherapy have commenced across a range of cancers, and the combination of PARP inhibitors and PSMA radioligand therapy has also entered clinical trials (NCT03874884). In this context, combination studies of radionuclide therapy with inhibitors of DNA repair may provide an enhanced therapeutic response, particularly when germline or somatic mutations of DNA repair genes are present.

\section{SUMMARY}

Pharmacogenomics may play a vital role in assessing patients who undergo radionuclide therapy, and prospective studies exploring germline and somatic mutations, as well as gene expression profiling and their relationship to response, are urgently required for prediction of efficacy and toxicity. We propose systematic inclusion of pharmacogenomics in prospective trials as a key strategy to inform future treatment strategies in patients undergoing radionuclide therapy.

\section{DISCLOSURE}

Andrew Scott is supported by NHMRC investigator fellowship 1177837. No other potential conflict of interest relevant to this article was reported.

\section{REFERENCES}

1. Wheeler HE, Maitland ML, Dolan ME, Cox NJ, Ratain MJ. Cancer pharmacogenomics: strategies and challenges. Nat Rev Genet. 2013;14:23-34

2. Huang RX, Zhou PK. DNA damage response signaling pathways and targets for radiotherapy sensitization in cancer. Signal Transduct Target Ther. 2020;5:60.

3. Bodei L, Schöder H, Baum RP, et al. Molecular profiling of neuroendocrine tumours to predict response and toxicity to peptide receptor radionuclide therapy. Lancet Oncol. 2020;21:e431-e443.

4. Ho AL, Grewal RK, Leboeuf R, et al. Selumetinib-enhanced radioiodine uptake in advanced thyroid cancer. N Engl J Med. 2013;368:623-632.

5. Bodei L, Kidd MS, Singh A, et al. PRRT genomic signature in blood for prediction of ${ }^{177}$ Lu-octreotate efficacy. Eur J Nucl Med Mol Imaging. 2018;45:1155-1169.

6. Baum R, Prasad V, Singh A, Zhang J, Sartor A, Kulkarni H. Germline gene variants and therapy response in patients referred for radioligand therapy with ${ }^{177} \mathrm{Lu}$-PSMA: a huge step towards pharmacogenomics in theranostics of prostate cancer [abstract]. J Nucl Med. 2020;61(suppl 1):1272.

7. Kratochwil C, Giesel FL, Heussel CP, et al. Patients resistant against PSMA-targeting $\alpha$-radiation therapy often harbour mutations in DNA damage-repair-associated genes. J Nucl Med. 2020;61:683-688.

8. Ahmadzadehfar H, Gaertner F, Lossin PS, Schwarz B, Essler M. BRCA2 mutation as a possible cause of poor response to ${ }^{177}$ Lu-PSMA therapy. Clin Nucl Med. 2018;43:609-610.

9. Paganelli G, Severi S, Sansovini M, et al. Circulating androgen receptor gene amplification and resistance to ${ }^{177}$ Lu-PSMA-617 in patients with metastatic castrate-resistant prostate cancer: results of a phase 2 trial [abstract]. Eur J Nucl Med Mol Imaging. 2020;47(suppl 1):S487. 